\title{
Fluvastatin Induces Apoptosis in Primary and Transformed Mast Cells ${ }^{[\mathbf{s}}$
}

\author{
Patrick A. Paez, Motunrayo Kolawole, Marcela T. Taruselli, Siddarth Ajith, \\ Jordan M. Dailey, Sydney A. Kee, (1)Tamara T. Haque, Brian O. Barnstein, \\ Jamie Josephine Avila McLeod, 애eather L. Caslin, Kasalina N. Kiwanuka, \\ Yoshihiro Fukuoka, Quang T. Le, Lawrence B. Schwartz, David B. Straus, David A. Gewirtz, \\ Rebecca K. Martin, and John J. Ryan \\ Departments of Biology (P.A.P., E.M.K., M.T.T., S.A., J.M.D., S.A.K., T.T.H., B.O.B., J.J.A.M., H.L.C., K.N.K., Y.F., D. B.S., \\ J.J.R.), Internal Medicine (Q.T.L., L.B.S.), Pharmacology and Toxicology (D.A.G.), and Microbiology and Immunology (R.K.M.), \\ Virginia Commonwealth University, Richmond, Virginia
}

Received November 25, 2019; accepted April 1, 2020

\begin{abstract}
Statin drugs are widely employed in the clinic to reduce serum cholesterol. Because of their hydroxymethylglutaryl coenzyme A reductase antagonism, statins also reduce isoprenyl lipids necessary for the membrane anchorage and signaling of small G-proteins in the Ras superfamily. We previously found that statins suppress immunoglobulin $\mathrm{E}$ (lgE)-mediated mast cell activation, suggesting these drugs might be useful in treating allergic disease. Although IgE-induced function is critical to allergic inflammation, mast cell proliferation and survival also impact atopic disease and mast cell neoplasia. In this study, we describe fluvastatin-mediated apoptosis in primary and transformed mast cells. An $\mathrm{IC}_{50}$ was achieved between 0.8 and $3.5 \mu \mathrm{M}$ in both cell types, concentrations similar to the reported fluvastatin serum $\mathrm{C}_{\max }$ value. Apoptosis was correlated with reduced stem cell factor (SCF)-mediated signal transduction, mitochondrial dysfunction, and caspase activation.
\end{abstract}

Complementing these data, we found that p53 deficiency or $\mathrm{Bcl}-2$ overexpression reduced fluvastatin-induced apoptosis. We also noted evidence of cytoprotective autophagy in primary mast cells treated with fluvastatin. Finally, we found that intraperitoneal fluvastatin treatment reduced peritoneal mast cell numbers in vivo. These findings offer insight into the mechanisms of mast cell survival and support the possible utility of statins in mast cell-associated allergic and neoplastic diseases.

\section{SIGNIFICANCE STATEMENT}

Fluvastatin, a statin drug used to lower cholesterol, induces apoptosis in primary and transformed mast cells by antagonizing protein isoprenylation, effectively inhibiting stem cell factor (SCF)-induced survival signals. This drug may be an effective means of suppressing mast cell survival.

\section{Introduction}

Statin drugs were approved by the Food and Drug Administration in the early 1990s to combat the rising incidence of hypercholesterolemia in the United States. These drugs reduce serum cholesterol and low-density lipoproteins, which have been linked to coronary heart disease and stroke (Khush and Waters, 2006). However, statins also have anti-inflammatory and antineoplastic effects that broaden their possible clinical utility. The intended pharmacological mechanism of statins is hydroxymethylglutaryl coenzyme A reductase (HMGCR)

This work was supported by grants from the National Institutes of Health [R01AI138495 and R21AI138494 (to J.J.R)].

https://doi.org/10.1124/jpet.119.264234.

S This article has supplemental material available at jpet.aspetjournals.org. antagonism. HMGCR is the rate-limiting step in cholesterol synthesis, yielding mevalonic acid. Although mevalonic acid is processed to cholesterol, intermediate and side reactions in this cascade also generate the isoprenoids farnesyl pyrophosphate (FPP) and geranylgeranyl pyrophosphate (GGPP). Isoprenylation, the process of conjugating FPP and GGPP to substrates, is required for subcellular localization of many proteins, particularly the Ras superfamily of small G-proteins. Because Ras members are critical to proliferation, migration, and cytokine production, there is great interest in determining if statins can be an efficacious therapy for inflammatory diseases in which G-protein pathways figure prominently (Takai et al., 2001; Zhang et al., 2013).

Epidemiologic studies have noted that asthmatic patients prescribed statins experienced fewer emergency department

ABBREVIATIONS: AKT, protein kinase functioning in survival and $\mathrm{PI} 3 \mathrm{~K}$ signaling; $\mathrm{Bcl}-2$, B-cell lymphoma 2, anti-apoptotic signaling; $\mathrm{BMMC}$, bone marrow-derived mast cells; cRPMI, complete RPMI 1640; $\mathrm{Di}\left(\mathrm{OC}_{6}\right)_{3}, 3,3^{\prime}$-dihexyloxacarboncyanine iodide; ERK, mitogen-activated protein kinase; FceRI, High affinity IgE receptor; FPP, farnesyl pyrophosphate; G2/M, Gap two/ mitosis of the cell cycle; GGPP, geranylgeranyl pyrophosphate; GGT, geranylgeranyl transferase; HMGCR, hydroxymethylglutaryl coenzyme A reductase; IgE, immunoglobulin E; IL-3, interleukin 3; KO, knockout; LC3, light chain-associated microtubule protein; MAPK, mitogen-activated protein kinase; $p$, Phosphorlated; PI-DNA, propidium iodide DNA; PMC, peritoneal mast cell; Ras, a small GTP binding protein; SCF, stem cell factor; Tg, transgenic expressed; WT, wild type; ZA, zaragozic acid A. 
visits for their allergic conditions as compared with their counterparts (Alexeeff et al., 2007; Huang et al., 2011; Wang et al., 2018). These reports prompted us to study statin effects on mast cells during the allergic response. Mast cell activation through the high-affinity immunoglobulin E (IgE) receptor (FceRI) is critical to allergic pathology. Antigen-mediated crosslinking of $\operatorname{IgE}$ bound to FceRI induces release of preformed granules containing proteases and histamine, followed by secretion of arachidonic acid metabolites and cytokines. Collectively, these mediators induce cellular infiltration, vasodilation, and bronchoconstriction, leading to edema, dyspnea, tissue damage, and even systemic shock (Metcalfe et al., 2009, 2016). We found that statins are powerful suppressors of IgE-mediated mast cell activation. Of the statins tested, fluvastatin was most effective in suppressing mast cell function in vitro and in vivo (Kolawole et al., 2016), with effects that could be tied to loss of GGPP (Fujimoto et al., 2009; Kolawole et al., 2016).

In addition to activation, mast cell numbers increase in allergic disease. This can be due to hyperplasia and recruitment of mast cell precursors (Elieh Ali Komi and Bjermer, 2019). Reducing mast cell numbers in diseases such as asthma and atopic dermatitis, in addition to inhibiting inflammatory function, could be a beneficial approach to treatment. Separate from their role in allergic disease, mast cells can form neoplasms. Aberrant mast cell proliferation ranges from slowgrowing localized mastocytomas to systemic mastocytosis and aggressive mast cell leukemias. These have a very high incidence $(>90 \%)$ of CD117 (c-Kit) D816V mutation that drives survival and proliferation (Castells and Butterfield, 2019). Mutant c-Kit is the target of clinical interventions but shows a high rate of drug resistance (Jin et al., 2014). Therefore, therapies limiting D816V c-Kit signaling could be useful in mast cell neoplasias.

Although short-term ( $<24$ hour) fluvastatin exposure suppresses mast cell function, we noted that fluvastatin induces mast cell apoptosis within 4 days of culture. This report details the mechanism of fluvastatin-induced apoptosis, which we find occurs in both primary and transformed mast cells. We also noted evidence of cytoprotective autophagy that delays fluvastatin-mediated cell death. We provide in vivo support for these effects and offer mechanistic insights that support the possible clinical use of statins in mast cell-associated diseases.

\section{Materials and Methods}

Reagents and Cell Lines. Murine interleukin 3 (IL-3) and stem cell factor (SCF) were purchased from Shenandoah Biotechnology, Inc. (Warwick, PA). Fluvastatin was purchased from Biovision, Inc. (Milpitas, CA). Mevalonic acid, SC66 an Akt inhibitor, bafilomycin A, chloroquine diphosphate, and Akti-1/2 (Akt inhibitors) were purchased from Millipore Sigma (Burlington, MA). Geranylgeranyl pyrophosphate (GGPP) and farnesyl pyrophosphate (FPP) were purchased from Echelon Biosciences (Salt Lake City, UT). Zaragozic acid A (ZA), geranylgeranyl transferase (GGT) inhibitor 286, and farnesyl transferase inhibitor II were purchased from Cayman Chemical (Ann Arbor, MI). FR180204, SCG772984, and ravoxertinib were purchased from Medchem Express LLC (Monmouth Junction, NJ). The 10-DEBC HCl was purchased from Tocris Biosciences (Minneapolis, MN). The mast cells lines P815 and spontaneous peritoneal derived mast cell line (PDMC-1) were the kind gift from Dr. Thomas Huff (Virginia Commonwealth University). RBL-2H3 cells were purchased from American Type Culture Collection (Rockville, MD).

Mice. C57BL/6J, 129/SvImJ, and p53 knockout (KO) mice were purchased from the Jackson Laboratory (Bar Harbor, ME) and bred in our facility. Bone marrow from $\mathrm{H} 2 \mathrm{~K}$ Bcl2-Tg mice was the kind gift of Kevin Bunting (Emory University). Mice were maintained at Virginia Commonwealth University facilities in accordance with Institutional Animal Care and Use Committee guidelines and euthanasia criteria.

Bone Marrow-Derived Mast Cell and Peritoneal Mast Cell Cultures. Bone marrow-derived mast cells (BMMC) were derived from bone marrow extracted from femurs and tibias that was depleted of red blood cells and placed in complete RPMI 1640 (cRPMI) medium (Invitrogen Life Technologies, Carlsbad, CA) containing 10\% heatinactivated FBS, $2 \mathrm{mM}$ l-glutamine, $10 \mathrm{U} / \mathrm{ml}$ penicillin, $10 \mu \mathrm{g} / \mathrm{ml}$ streptomycin, $1 \mathrm{mM}$ sodium pyruvate, and $10 \mathrm{mM}$ HEPES (Biofluids, Rockville, MD), supplemented with SCF-containing supernatant from baby hamster kidney-mouse kit ligand (BHK-MKL) cells and IL-3 from a transfected B-cell lymphoma cell line WEHI-3B cells for 21-28 days. Final concentrations of SCF and IL-3 were adjusted to 15 and $5 \mathrm{ng} / \mathrm{ml}$, respectively, as measured by ELISA. Peritoneal mast cells (PMC) were expanded from peritoneal lavage cells cultured as described (Caslin et al., 2018) by using the same media as stated for BMMC, with selection against adherent cells for 7-14 days. At the time of use, BMMC and PMC were $>95 \%$ mast cells, based on dual staining for FceRI and c-Kit, as assessed by flow cytometry.

Human Mast Cells. Mast cell isolation from human skin was performed as previously described (Kambe et al., 2001). Briefly, mast cells were dispersed from the skin, enriched, and cultured in X-VIVO 15 medium (Lonza, Walkersville, MD) containing recombinant human stem cell factor $(100 \mathrm{ng} / \mathrm{ml}$; Swedish Orphan Biovitrum, Stockholm, Sweden). Without other cytokines or serum, non-mast cell populations do not survive, leaving mast cells of greater than $98 \%$ purity after approximately 3 weeks.

Flow Cytometric Analysis. All flow cytometric analysis was performed using a BD FACSCaliber or BD FACSCelesta (BD Biosciences, San Jose, CA). Cell surface staining was conducted using antibodies against the following mouse proteins: CD117 (c-KIT), CD25, CD2, and CD16/CD32 (eBiosciences, Waltham, MA). For propidium iodide DNA (PI-DNA) staining, cells (1 to $2 \times 10^{5}$ per sample) were placed in $200 \mu$ l fixation buffer (36\% ethanol, $12.8 \%$ fetal calf serum, and $50.8 \% 1 \times \mathrm{PBS}$ ) for at least 4 hours at $4^{\circ} \mathrm{C}$, washed with PBS, and resuspended in $200 \mu \mathrm{l}$ staining buffer (PBS containing $100 \mu \mathrm{g} / \mathrm{ml}$ RNase A and $50 \mu \mathrm{g} / \mathrm{ml}$ PI) for 2 hours at room temperature in the dark. For staining with 3,3'-dihexyloxacarboncyanine iodide $\left[\mathrm{Di}\left(\mathrm{OC}_{6}\right)_{3}\right.$; Enzo Life Sciences, Farmingdale, $\left.\mathrm{NY}\right]$, a final concentration of $1 \mathrm{nM} \mathrm{Di}\left(\mathrm{OC}_{6}\right)_{3}$ was added to culture media for 30 minutes at $37^{\circ} \mathrm{C}$ in the dark prior to flow cytometry analysis. Caspase 7/9 activity DEVD assays were performed according to the manufacturer's instructions (Thermo Fisher, Waltham, MA).

Western Blotting. Western blots were conducted using standard protocols. Nitrocellulose membrane $(0.45 \mu \mathrm{M})$ was purchased from Bio-Rad (Hercules, CA). The following primary antibodies were used at a 1:1000 dilution of the stock concentration: light chain-associated microtubule protein (LC3) I/II, p62, glyceraldehyde-3-phosphate dehydrogenase (GAPDH), ERK1/2, phospho-ERK1/2, AKT, phosphoAKTser473, p85, phospho-p85 (Cell Signaling, Danvers, MO). Western blot membranes were blocked with Tris-buffered saline-bovine serum albumin and incubated with primary antibodies for 1-3 days in Tris-buffered saline/0.1\% Tween $(0.1 \%)$ and $0.5 \%$ bovine serum albumin blocker, all from Thermo Fisher. Western blots were analyzed using infrared-conjugated secondary antibodies on an Odyssey Imaging System (LI-COR, Lincoln, NE).

Fluvastatin Use In Vivo. Female C57BL/6J mice received $10 \mathrm{mg} / \mathrm{kg}$ fluvastatin or vehicle (DMSO diluted in PBS to match fluvastatin solvent) daily by intraperitoneal injection for 4 days. Mice were sacrificed, and peritoneal lavage was harvested and analyzed for mast cells by staining for CD45+ cells and gating on FceRI+/c-Kit+ cells. Cross sections of small 
intestine were also analyzed by histology, using staining with pinacyanol erythosinate to identify mast cells.

Statistics. Data shown in each figure are the mean \pm S.D. Graphical and statistical analyses were conducted on GraphPad Prism 7. For comparisons of two samples, a Student's $t$ test was applied unless otherwise stated. ANOVA analysis with Bonferroni post hoc testing was used to compare three or more sample sets. Significance was assigned as follows: $P \leq 0.05^{*}, P \leq 0.01^{* *}, P \leq 0.001$ $* * *$, and $P \leq 0.0001^{* * * * *}$.

\section{Results}

Fluvastatin Induces Mast Cell Apoptosis. While investigating the ability of fluvastatin to suppress IgE-mediated mast cell activation, we found that apoptosis occurred after culturing cells with the drug for more than 2 days. Figure $1 \mathrm{~A}$ shows that approximately $60 \%$ of C57BL/6J BMMC were apoptotic after 4 days of culture in $1 \mu \mathrm{M}$ fluvastatin. A dose response of fluvastatin was conducted at 96 hours, which found that nearly all cells exhibited DNA fragmentation at concentrations of $5 \mu \mathrm{M}$ or greater (Fig. 1B). In addition to DNA fragmentation assessed by PI-DNA staining, we also noted a significant increase in caspase-3/7 activation (Fig. 1C).

Because we previously found that mast cells from the 129S1/ SvImJ background were fluvastatin-resistant (Kolawole et al., 2016), we compared the $\mathrm{IC}_{50}$ for apoptosis of BMMC from this strain to C57BL/6J. As shown in Fig. 1D, C57BL/6J BMMC exhibited an $\mathrm{IC}_{50}$ of $0.8 \mu \mathrm{M}$, whereas 129S1/SvImJ BMMC were threefold more resistant. Both values are within the 2.5 $\mu \mathrm{M}$ serum $\mathrm{C}_{\max }$ of fluvastatin in patients taking the drug (Siekmeier et al., 2001). By comparison, atorvastatin and simvastatin induced C57BL/6 BMMC apoptosis at higher concentrations of 20 and $6.5 \mu \mathrm{M}$, respectively (Supplemental Fig. 1). Interestingly, BMMC treated with fluvastatin undergo apoptosis with no discernable cell cycle arrest, even in the 24 hours preceding detectable apoptosis. Instead, all phases of the cell cycle were reduced by fluvastatin, with only the apoptotic fraction increasing (Supplemental Fig. 2).

We corroborated the BMMC data with similar testing of C57BL/6J PMC. These cultures showed a greater sensitivity to fluvastatin than BMMC, with an $\mathrm{IC}_{50}$ of approximately 0.2 $\mu \mathrm{M}$ and $90 \%$ apoptosis at a $0.5-\mu \mathrm{M}$ dose (Fig. $1 \mathrm{E}$ ). Furthermore, human skin-derived mast cells from three donors also showed varying degrees of apoptosis after 4 days of fluvastatin treatment (Fig. 1F). Human mast cells appeared to be more resistant than BMMC or PMC, with consistent apoptosis detected at $10 \mu \mathrm{M}$ of fluvastatin. These data support the conclusion that fluvastatin induces apoptosis of primary mouse and human mast cells.

Fluvastatin-Induced Apoptosis Targets the Geranylgeranyl Lipid Pathway. A simplified depiction of cholesterol and isoprenoid biosynthesis is shown in Fig. 2A. To determine the mechanism by which fluvastatin induces apoptosis, we first examined the effect of cholesterol blockade with ZA, which antagonizes squalene synthase, a reaction downstream from the HMGCR statin target and proximal to

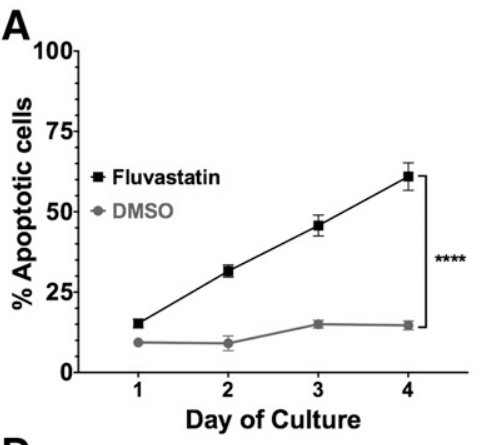

D

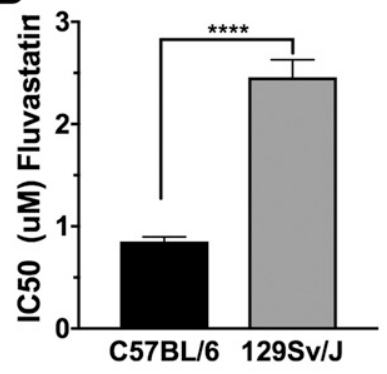

B

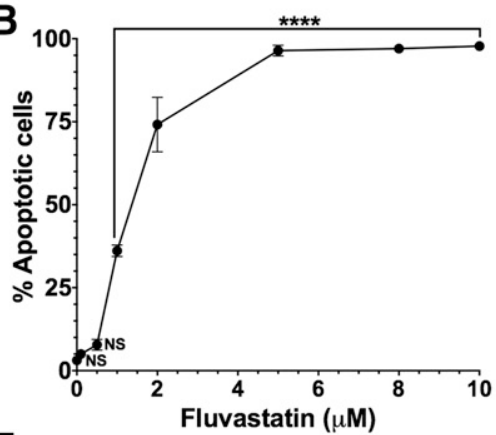

E

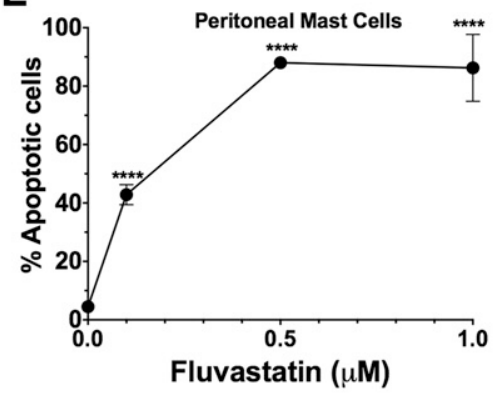

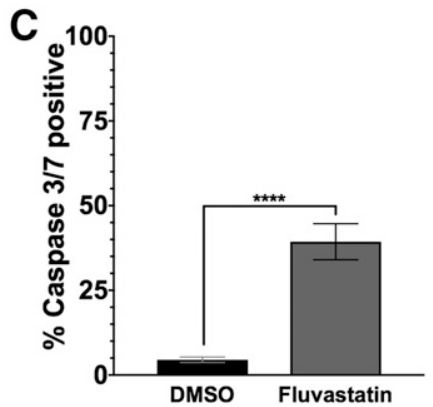

F

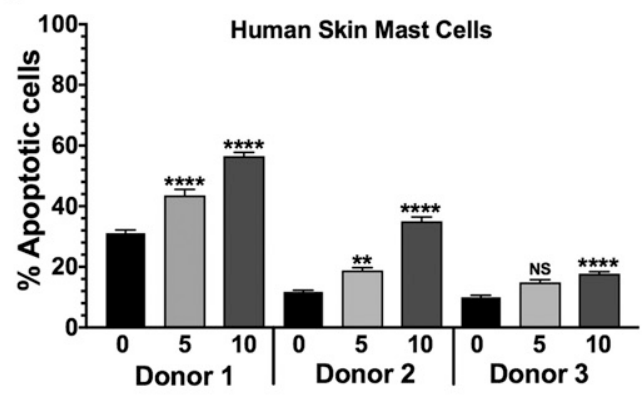

Fluvastatin $(\mu \mathrm{M})$

Fig. 1. Fluvastatin induces apoptosis in mouse BMMC and PMC. (A) C57BL/6 BMMC were cultured for the indicated times in $1 \mu M$ of fluvastatin, and apoptosis was assessed by PI-DNA staining of subdiploid DNA, as measured by flow cytometry. $P$ values were calculated by comparing fluvastatin vs. DMSO paired time points by two-way ANOVA. (B) BMMC were cultured for 4 days in the indicated fluvastatin concentrations. Apoptosis was measured by PI-DNA staining. $P$ values were calculated by comparing fluvastatin to the DMSO control by two-way ANOVA. (C) BMMC were cultured as in (A) for 4 days, and Caspase-3/7 activity was assessed by cleavage of fluorescent substrate and flow cytometry. (D) BMMC from the indicated strains were cultured in increasing concentrations of fluvastatin for 4 days, and IC $_{50}$ values were determined. (E) Primary PMC were cultured for 4 days in the indicated fluvastatin concentrations, and apoptosis was assessed by PI-DNA staining. (F) Human skin-derived mast cells were cultured for 4 days in the indicated concentrations of fluvastatin, and apoptosis was assessed by PI-DNA staining. Concentrations of "0" indicate DMSO controls in each figure, which were matched to the highest DMSO concentration present in fluvastatin-treated cells. Data shown for all figures are from six samples/point from one of at least three experiments. Not significant (NS), $P \leq 0.05 *, P \leq 0.01 * *, P \leq 0.001 * * *$, and $P \leq 0.0001^{* * * *}$ by ANOVA. 
cholesterol. As shown in Fig. 2B, ZA treatment had no effect on BMMC survival and did not enhance fluvastatin-induced cell death. By contrast, adding mevalonic acid, the HMGCR product, reversed the effects of fluvastatin (Fig. 2C). This indicates that fluvastatin-induced death is not an off-target drug effect. The 15-carbon isoprenoid lipid farnesyl pyrophosphate (FPP) was unable to reverse apoptosis, but the 20-carbon geranylgeranyl pyrophosphate (GGPP) provided significant protection from fluvastatin effects (Fig. 2C). These data were complemented by using farnesyl transferase or GGT inhibitors (Fig. 2D). Farnesyl transferase inhibition had no effect, whereas GGT blockade mimicked fluvastatin effects. Collectively, these data support the hypothesis that fluvastatininduced apoptosis results from HMGCR blockade that reduces downstream geranylgeranyl pyrophosphate.

Fluvastatin Decreases SCF-Mediated Survival Signals in BMMC. Isoprenoid lipids such as GGPP are critical for subcellular localization of small $G$ proteins in the Ras superfamily, which control activation of MAPK and other pathways (Wang and Casey, 2016). Hence, we suspected that statin-mediated GGPP reduction induced apoptosis by inhibiting growth factor signals. Because c-Kit function is critical for primary and neoplastic mast cell survival, we investigated how fluvastatin affects c-Kit signaling. BMMC were cultured for 24 hours in cRPMI supplemented with SCF and IL-3 at $10 \mathrm{ng} / \mathrm{ml}$, plus either vehicle (DMSO) or fluvastatin $(1 \mu \mathrm{M})$. Cells were then starved of IL-3+SCF for 4 hours prior to SCF restimulation. Western blots for phosphorylated pERK1/2 and pAKT were performed. A representative blot and quantification of pERK1/2:total ERK1/2 ratio, measured as fold increase from the unstimulated group, showed fluvastatin significantly reduced ERK phosphorylation (Fig. 3, A and B). By comparison, SCF-mediated AKT phosphorylation was unaffected. To further test the correlation of decreased ERK activity with apoptosis, we determined the effect of ERK blockade. As shown in Fig. 3C, three ERK inhibitors mirrored fluvastatin effects, inducing BMMC apoptosis. These data support the theory that fluvastatin effects on GGPP suppress the c-KitRas-ERK cascade, resulting in mast cell apoptosis.

Apoptosis is Dependent on p53 Expression and Mitochondrial Stability. Loss of c-Kit signaling can elicit p53 expression, which has been shown to occur in neoplastic mast cells expressing oncogenic c-Kit (Dos Santos et al., 2013; Bandara et al., 2018). Therefore, we measured fluvastatininduced apoptosis in p53 KO BMMC and their backgroundand age-matched wild-type (WT; C57BL/6) counterparts. BMMC were cultured in either vehicle (DMSO) or fluvastatin $(10 \mu \mathrm{M})$ in cRPMI with IL-3 and SCF at $10 \mathrm{ng} / \mathrm{ml}$ over a 72-hour time course. We found that p53 KO BMMC demonstrated significantly less fluvastatin-induced apoptosis than WT cells (Fig. 4A). Because p53 activation can induce mitochondrial damage, and statins have been shown to induce mitochondrial dysfunction (Ramachandran and Wierzbicki, 2017), we determined the effect of overexpressing the antiapoptotic mitochondrial protein Bcl-2. We cultured age- and strain-matched Bcl-2 transgenic and C57BL/6 WT BMMC with fluvastatin over a 72-hour time course and found that Bcl-2 overexpression significantly protected mast cells from apoptosis (Fig. 4B). These results indicate a role for the p53mitocondrial pathway in fluvastatin-mediated apoptosis, perhaps because of decreased c-Kit survival signals that can be compensated by overexpressing Bcl-2.

Fluvastatin Induces Cytoprotective Autophagy. In addition to apoptosis, statins or growth factor blockade can induce autophagy (Parikh et al., 2010; Zhang et al., 2013). One measure of autophagy is the accumulation of cleaved LC3 II and degradation of ubiquitin-binding protein p62, as detected by Western blotting. Because mast cells undergo constitutive autophagy (Ushio et al., 2011), we used chloroquine to raise lysosomal pH and inhibit LC3 II and p62 degradation during autophagy. Autophagy inhibition, alone and in combination with fluvastatin treatment, demonstrated changes from baseline autophagy via measurement of LC3 II and p62 accumulation. As shown in Fig. 5, A and B, fluvastatin significantly increased the accumulation of LC3 II and p62 in the presence of chloroquine. We also noted a modest increase in LC3 I in the presence of fluvastatin $(P<0.05)$, although the importance of this change is unclear. Thus, fluvastatin appears to increase mast cell autophagy. To determine how autophagy affects fluvastatin-induced apoptosis, we blocked autophagy with chloroquine or bafilomycin A and measured subdiploid DNA content. BMMC treated for 48 hours with fluvastatin showed modest but significant cell death at this early time point that was greatly enhanced by chloroquine or bafilomycin
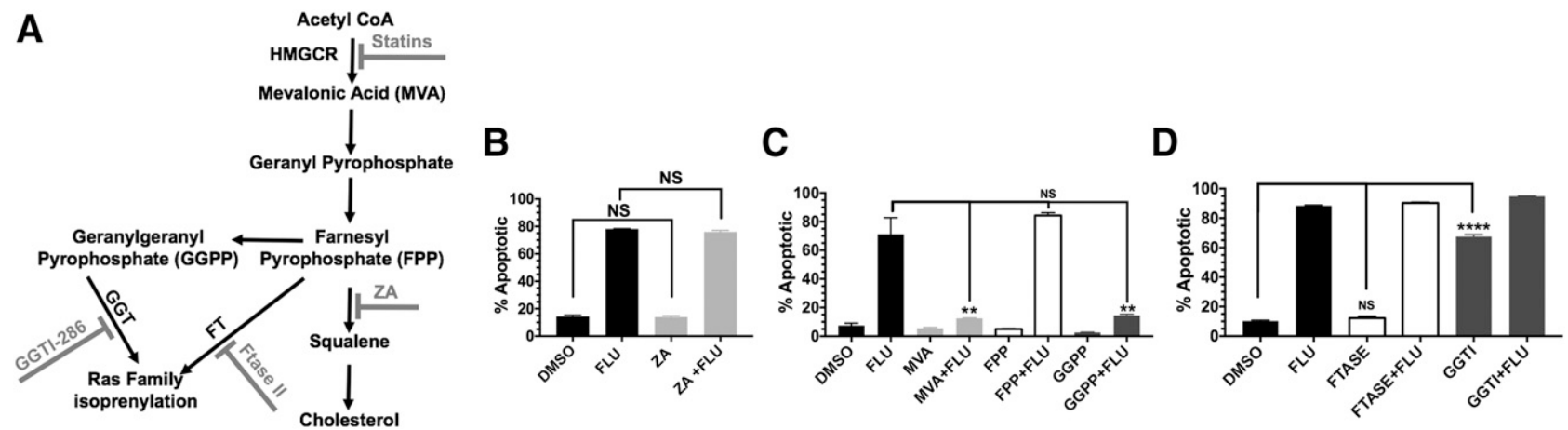

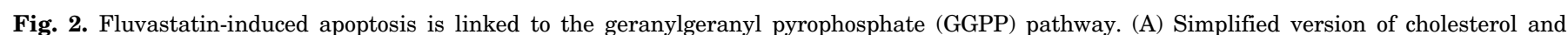

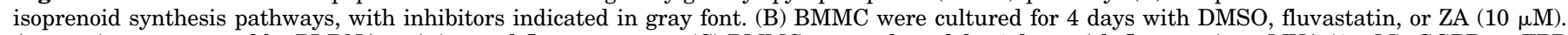

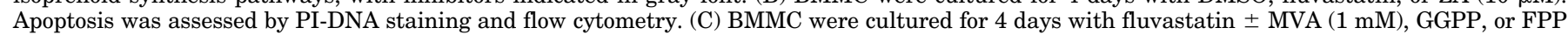

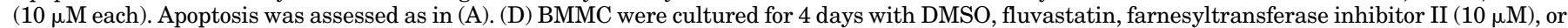

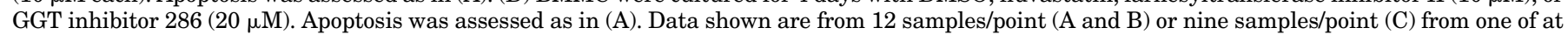

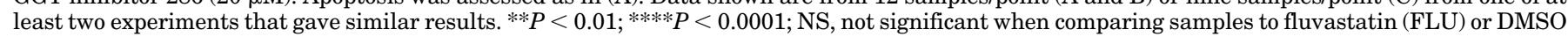
controls as indicated, using one-way ANOVA. MVA, mevalonic acid. 

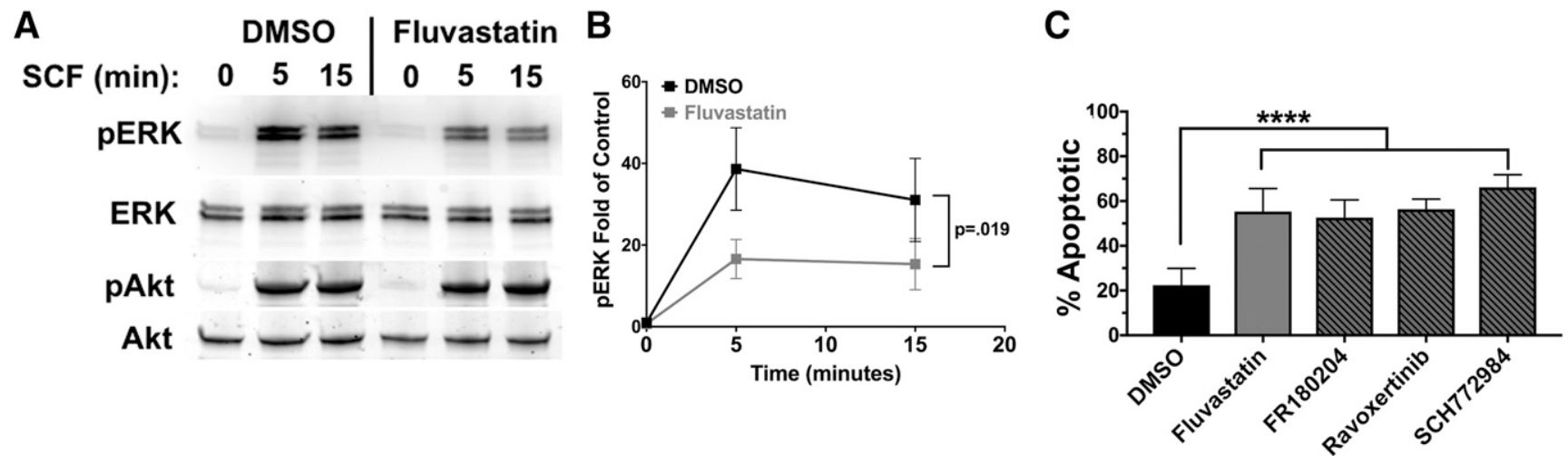

Fig. 3. Fluvastatin inhibits SCF-mediated ERK phosphorylation. (A and B) BMMC were cultured for 24 hours with DMSO or fluvastatin ( $1 \mu M$ ) prior to stimulation with $200 \mathrm{ng} / \mathrm{ml}$ SCF for the indicated times. Image in (A) shows example Western blot data; (B) shows results from three populations. (C) BMMC were cultured for 4 days with fluvastatin $(2.5 \mu \mathrm{M})$ or the indicated ERK inhibitors $(10,10$, and $5 \mu \mathrm{M}$, respectively). Data are from three populations. ${ }^{* * * *} P<0.0001$ by ANOVA.

A (Fig. 5C). These data suggest that fluvastatin induces cytoprotective autophagy that delays mast cell apoptosis.

Fluvastatin Suppresses Mast Cell Survival In Vivo. To examine fluvastatin effects on mast cell survival in vivo, mice were injected with fluvastatin $(10 \mathrm{mg} / \mathrm{kg})$ or vehicle (DMSO diluted in PBS) daily for 4 days via intraperitoneal route. As shown in Fig. 6, fluvastatin did not reduce total peritoneal cells but greatly reduced the proportion and total number of mast cells found in peritoneal lavage. Because we have previously found fluvastatin inhibits mast cell migration to factors such as SCF (Kolawole et al., 2016), it seems unlikely that this loss of mast cells is due to emigration to other sites. For example, we noted no increase in small intestine mast cell numbers (data not shown). These data support the hypothesis that fluvastatin suppresses mast cell survival in vivo.

Fluvastatin Induces Apoptosis in Transformed Mast Cells. Mastocytomas have a high incidence of c-Kit mutation that promotes their proliferation and survival (Carter et al., 2014). Because we found that fluvastatin inhibits c-Kit signaling in BMMC, we next determined if these effects extended to neoplastic mast cells. Mouse spontaneous peritoneal derived mast cell line (PDMC-1) and P815 and rat basophil leukemia (RBL-2H3) mastocytoma cells were cultured in vehicle (DMSO) or fluvastatin at a range of concentrations for 96 hours and were analyzed with PI-DNA staining. This yielded $\mathrm{IC}_{50}$ values of $1.5-3.5 \mu \mathrm{M}$, with nearly all cells killed above the $7-\mu \mathrm{M}$ dose (Fig. 7A and data not shown). Because of their intermediate $\mathrm{IC}_{50}$ and ease of culture, we continued analyzing the P815 cell line.
Unlike BMMC, P815 cells showed a significant loss of synthesis (S) phase and a G2 cell cycle arrest at the 24-hour time point preceding apoptosis (Fig. 7B). Because fluvastatininduced apoptosis was suppressed by Bcl-2 overexpression in BMMC, we investigated the effect of fluvastatin on mitochondrial stability in P815 cells. Cells were cultured for 48 hours in DMSO or fluvastatin and stained with $\mathrm{Di}\left(\mathrm{OC}_{6}\right)_{3}$, a dye with high affinity for the mitochondria. Decreased staining indicates loss of the trapped dye, consistent with mitochondrial membrane damage. $\mathrm{P} 815$ cells treated with fluvastatin exhibited diminished $\mathrm{Di}\left(\mathrm{OC}_{6}\right)_{3}$ staining compared with vehicle-treated cells (Fig. 7, C and D). Given this indication of mitochondrial damage, we next assessed the presence of active caspase 9, which can result from mitochondrial membrane instability. P815 cells treated for 24 hours with fluvastatin had increased active caspase 9 activity, as measured using a cleavable fluorescent caspase-9 peptide (Fig. 7E). These data further support the theory that fluvastatin induces apoptotic cell death via a mitochondrial pathway.

We also examined changes in c-Kit expression and signaling on P815 mastocytoma cells. When gating on live cells, we found that c-Kit surface expression increased from 12 to 48 hours of culture in fluvastatin (Fig. 8A). In contrast to increased c-Kit levels, constitutive phosphorylation of ERK and Akt was greatly diminished by fluvastatin treatment (Fig. 8B). To link these changes to survival, we tested the effects of ERK or Akt inhibitors. Although one of three ERK inhibitors induced P815 apoptosis when used at 5-10 $\mu \mathrm{M}$ concentration, all three Akt inhibitors elicited significant cell death (Fig. 8C). These data support the theory that
A

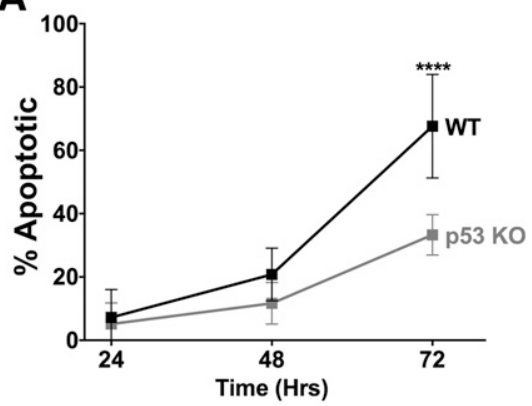

B

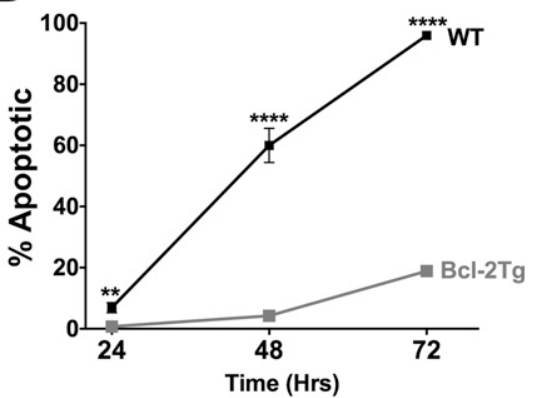

Fig. 4. Fluvastatin induces Bcl-2- and p53-dependent apoptosis. BMMC from C57BL/6, H-2 ${ }^{\mathrm{k}}$-p53 KO (A) or $\mathrm{Bcl} 2 \mathrm{Tg}(\mathrm{B})$ mice were cultured with Fluvastatin $(10 \mu \mathrm{M})$ for the indicated times. Apoptosis was determined by subdiploid DNA content measured by PI-DNA stain using flow cytometry. Data shown are mean \pm S.D. from six to nine samples from one of two separate experiments. Some error bars are too small to be shown. $* * P<$ $0.01 ; * * * * P<0.0001$ by ANOVA. 

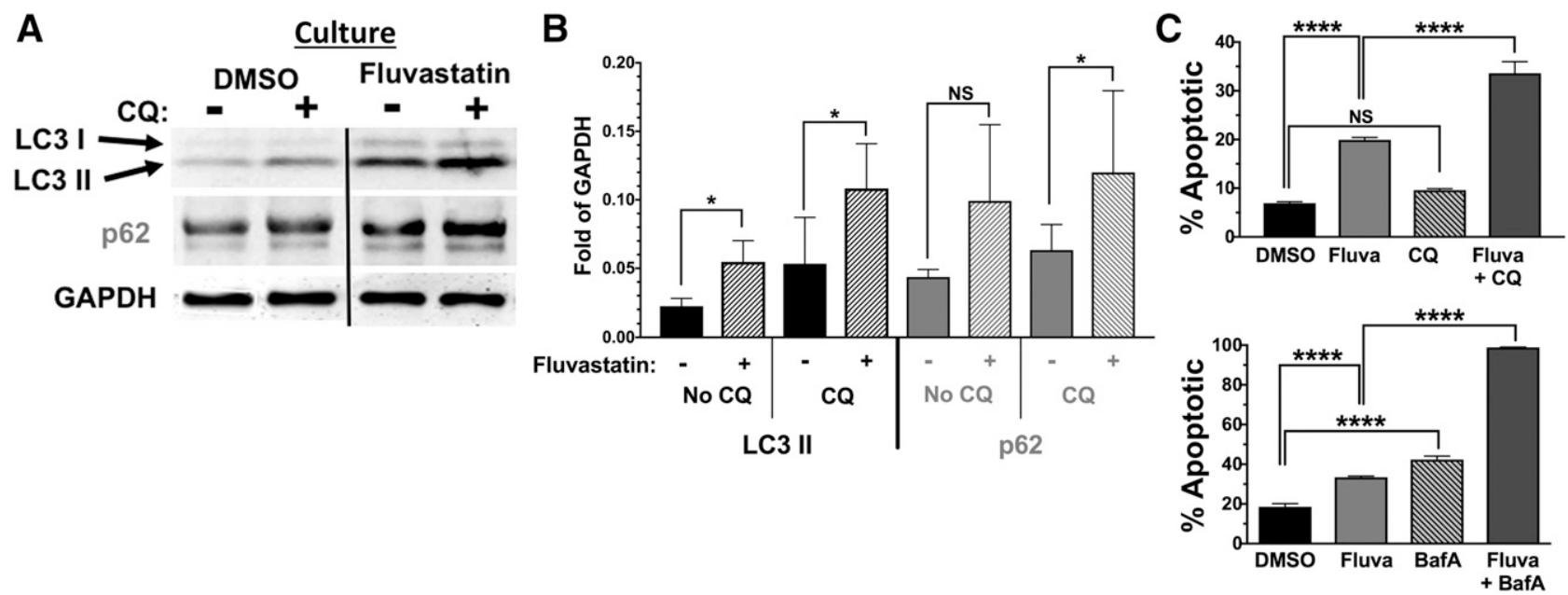

Fig. 5. Fluvastatin (Fluva) induces LC3 II and p62 accumulation in BMMC. (A) Example Western blot of BMMC cultured for 24 hours with DMSO or Fluva $(10 \mu \mathrm{M}) \pm$ chloroquine $(\mathrm{CQ})$, used to enhance LC3 II and p62 detection. (B) Summary data from three BMMC populations treated as in (A), using 5 or $10 \mu \mathrm{M}$ Fluva compared with matched DMSO samples. $* P<0.05$ by paired $t$ test comparing each Fluva-treated sample to the DMSO control. (C) C57BL/6 BMMC were cultured with vehicle (DMSO) or Fluva \pm CQ or bafilamycin A (BafA) for 48 hours, and apoptosis was measured by PI-DNA staining and flow cytometry. Data shown are from six to nine samples/point. $* * * * P<0.0001$ by ANOVA. GAPDH, glyceraldehyde-3-phosphate dehydrogenase.

fluvastatin-induced apoptosis is linked to loss of survival signals provided by constitutively active c-Kit in mastocytoma cells, with particular importance for the Akt pathway. P815 cells appear to transiently increase c-Kit expression, perhaps as a compensatory mechanism that delays cell death.

\section{Discussion}

Mast cells primarily defend against bacterial and parasitic infections. But in allergic disease, environmental antigens elicit pathologic mast cell activation when antigen aggregates receptor-bound IgE. There is an early and a late phase of mast cell activation that collectively cause vasodilation, capillary leakage, and recruitment and activation of other immune cells. The early phase of activation occurs when preformed granules containing histamine and proteases are released, causing increased vascular permeability and vasodilation. The late phase follows 4-6 hours later, elicited by cytokine and chemokine production (Metcalfe et al., 2009, 2016). In addition to cell activation, mast cell hyperplasia occurs in allergic and neoplastic diseases (Elieh Ali Komi and Bjermer, 2019); hence, reducing mast cell numbers is an important clinical goal.

Several studies have shown correlations between statin therapy and reduced emergency department visits by asthmatic/allergic patients (Alexeeff et al., 2007; Huang et al.,
2011; Wang et al., 2018). HMGCR antagonism by statins reduces farnesyl and geranylgeranyl pyrophosphate, isoprenoid lipids that are coupled to Ras superfamily proteins (Wang and Casey, 2016). The loss of isoprenylation yields small G-proteins without membrane anchorage, diminishing signal transduction downstream of ligand-receptor interaction. Mechanisms possibly explaining statin antiallergic effects include our recent demonstration that fluvastatin decreases MAPK and AKT signaling pathways activated by IgE crosslinking (Kolawole et al., 2016). In longer time courses, we noted that a significant proportion of BMMC died when treated with statins. Although atorvastatin and simvastatin induced significant apoptosis, they did so at relatively high concentrations; hence, we focused on fluvastatin. This was in keeping with our previous study of statin-mediated suppression of IgE signaling, in which fluvastatin was the most consistently effective of the statins tested (Kolawole et al., 2016). Because most patients use statins for longterm therapy, statin-induced apoptosis may be clinically relevant. This possibility prompted our studies of apoptosis and autophagy.

This work shows that primary mast cells treated with fluvastatin undergo a p53-dependent apoptosis but with no discernible cell cycle arrest. We noted selective loss of c-Kit-mediated signaling, with significant ERK blockade. This correlation between reduced ERK activation and apoptosis
A

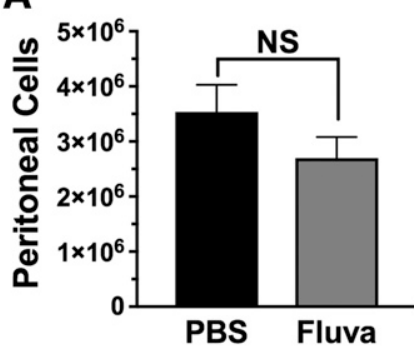

B

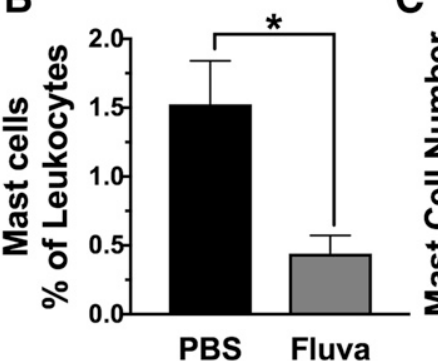

C

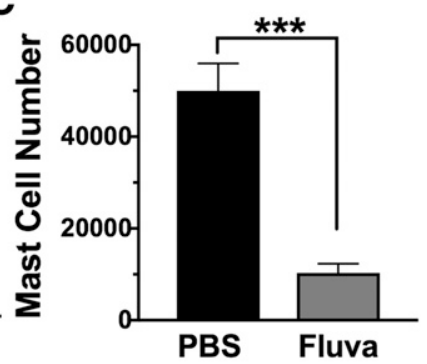

Fig. 6. Fluvastatin reduces peritoneal mast cell number in vivo. C57BL/6 mice (four to six per group) were injected with $10 \mathrm{mg} / \mathrm{kg}$ i.p. fluvastatin once daily for 4 days. Peritoneal lavage fluid was analyzed by flow cytometry to identify mast cells Figure A-C (FceRI+, c-Kit+). Not significant (NS), $P \leq 0.05 *, P \leq 0.01 * *$, $P \leq 0.001 * * *$, and $P \leq 0.0001^{* * * *}$ by $t$ test. 

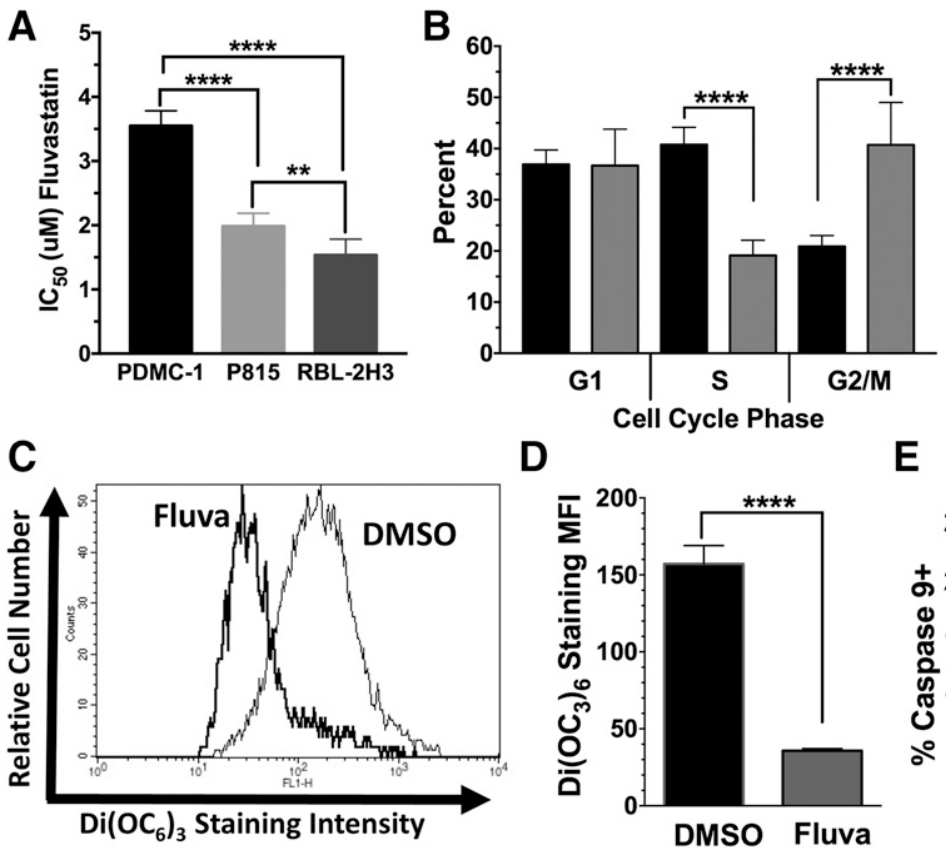

E

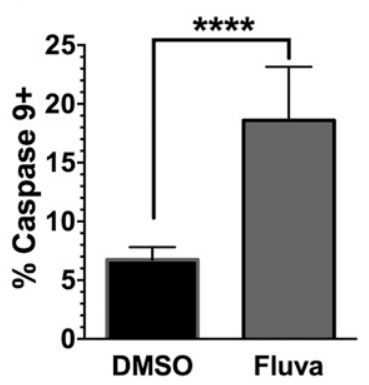

Fig. 7. Fluvastatin induces apoptosis in transformed mast cells. (A) The indicated mastocytoma cell lines (PDMC-1, P815, RBL-2H3) were cultured for 4 days in fluvastatin, and $\mathrm{IC}_{50}$ values were calculated as in Fig. 1. Data are means and S.D. of eight samples, with $P$ values calculated by one-way ANOVA. (B) P815 cells were cultured for 24 hours in fluvastatin $(10 \mu \mathrm{M})$, and cell cycle was measured using PI-DNA staining and flow cytometry. Data are means and S.D. of seven to nine samples from one of three representative experiments, with $P$ values calculated by $t$ test. (C and D) Representative histogram and summary data of P815 cells stained with $\mathrm{Di}\left(\mathrm{OC}_{6}\right)_{3}$ after 48 hours of culture with fluvastatin $(10 \mu \mathrm{M})$. (D) shows means and S.D. of six samples, with $P$ value calculated by $t$ test. (E) P815 cells were cultured for 48 hours, and Caspase-9 activity was assessed by cleavage of fluorescent substrate and flow cytometry. Data are means and S.D. of 12 samples from one of two representative experiments, with $P$ value calculated by t test. Fluvastatin, (Fluva); S, synthesis. $P \leq 0.05^{*}, P \leq 0.01^{* *}, P \leq 0.001^{* * *}$, and $P \leq 0.0001 * * * *$ is supported by the literature. MAPK proteins are involved in labeling proapoptotic Bcl-2 homology domain (BH3 only) proteins such as Bim for proteasome degradation in mast cells (Moller et al., 2005; Karlberg et al., 2010; Westerberg et al., 2012). Thus, decreased ERK/MAPK function is a plausible mechanism for apoptosis that should be overcome by Bcl2 overexpression, as we found in Fig. 4 . We therefore propose that fluvastatin-induced apoptosis is driven by loss of survival signals from c-Kit and perhaps other growth factor receptors.

The timing of apoptosis, which peaked at 4 days of fluvastatin treatment, appears to be due, in part, to cytoprotective autophagy. Blocking autophagy accelerated fluvastatinmediated cell death, suggesting that autophagy delays apoptosis. Interestingly, a recent study found that mast cells have a constant autophagic flux necessary for granule maintenance (Ushio et al., 2011), but how autophagy affects mast cell survival is poorly understood. Statins have been shown to alter autophagy and cause mitochondrial dysfunction (Parikh et al., 2010; Zhang et al., 2013). An apparent role for the mitochondrion, as evidenced by data from p53 KO and Bcl-2 Tg BMMC, offers a potential link to a form of autophagy called mitophagy. In this model, autophagy blockade causes the accumulation of damaged mitochondria that release reactive oxygen species and have decreased oxidative phosphorylation (Youle and Narendra, 2011; Roberts et al., 2016; Springer and Macleod, 2016). This combination of poor functioning and increased reactive oxygen species accelerates apoptosis, similar to the outcome we found. Although our data are consistent with mitophagy, further study is needed to determine if this is the specific form of autophagy occurring.

Fluvastatin injections for 4 days greatly reduced peritoneal mast cell numbers without significant change in the total

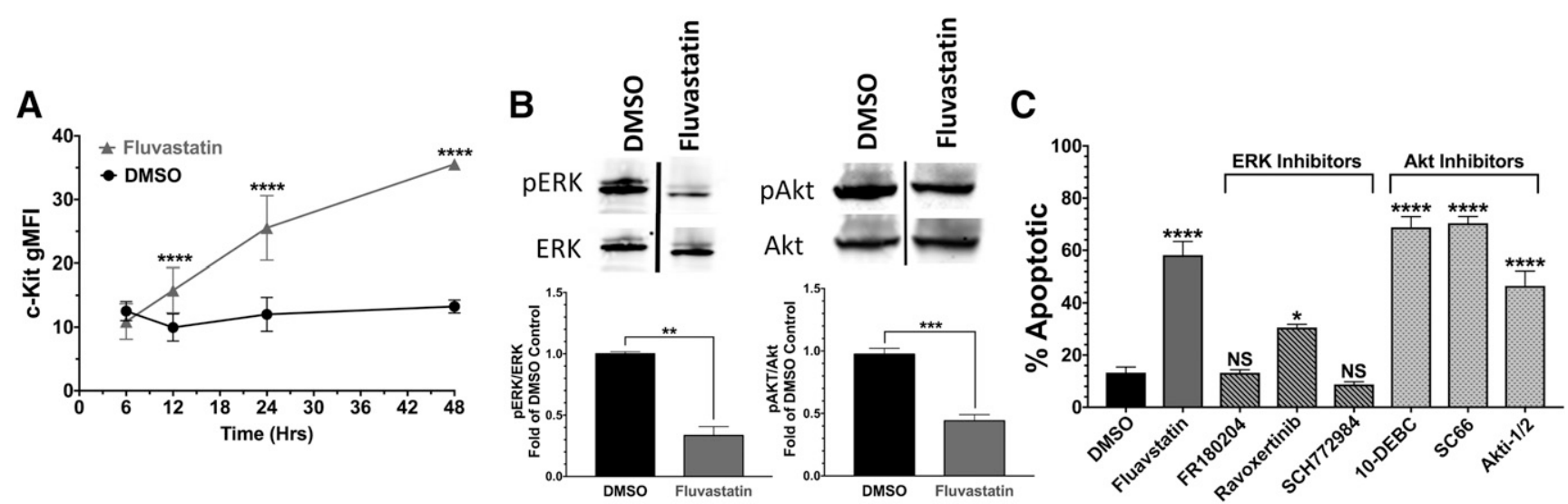

Fig. 8. Fluvastatin increases c-Kit expression but inhibits signaling in mastocytoma cells. (A) P815 cells were cultured for the indicated times in DMSO or fluvastatin, and c-Kit expression was measured by flow cytometry. (B) P815 cells were cultured for 24 hours in DMSO or fluvastatin (10 $\mu$ M), and lysates were probed for phosphorylated ( $\mathrm{p}$ ) and total ERK or AKT expression by Western blot. Bar charts show summary data from six to seven samples/ point. (C) P815 cells were cultured for 4 days with DMSO, fluvastatin $(10 \mu \mathrm{M})$, or the indicated ERK $(10,5,10 \mu \mathrm{M}$, respectively) or AKT (10 $\mu \mathrm{M})$ inhibitors, and apoptosis was measured by PI-DNA staining and flow cytometry Not significant (NS), $P \leq 0.05 *, P \leq 0.01 * *, P \leq 0.001 * * *$, and $P \leq$ $0.0001^{* * * *}$ by ANOVA (A and C) or $t$ test (B). 
peritoneal population. Because mast cells comprise $1 \%-3 \%$ of peritoneal cells (Morii et al., 2004), these data suggest that either mast cells are particularly sensitive to fluvastatinmediated death or perhaps other cell types are repopulated more quickly. Because we previously found that fluvastatin inhibits mast cell migration (Kolawole et al., 2016), it is unlikely that drug treatment caused significant emigration out of the peritoneum. Fluvastatin has been shown to reduce adhesion molecule expression on endothelial cells and monocytes (Xenos et al., 2005) and to suppress eosinophil and leukocyte adhesion (Niwa et al., 1996; Fischetti et al., 2004; Robinson et al., 2009). Therefore, it is unlikely that fluvastatin selectively induced mast cell adhesion leading to reduced recovery in peritoneal lavage fluid. These data support the theory that fluvastatin may reduce mast cell numbers in vivo, an effect with clinical utility in both atopic and neoplastic conditions that warrants further investigation.

Data from primary cells prompted interest in statin effects on transformed mast cells. The P815 model used in our study conforms to several aspects of the mast cell leukemia diagnosis, including expression of the mutant c-Kit receptor, which causes constitutive growth factorindependent proliferation (Tsujimura et al., 1994). There has been considerable research employing statin therapy with various cancer types, including those with mutant Kirsten rat Ras protein (K-Ras) or B-Raf serine/threonine protein kinase oncogene (Zhang et al., 2013). In particular, fluvastatin has been shown to be effective against myeloid leukemias in vitro and in vivo (Dai et al., 2007; Sassano et al., 2007; Calabro et al., 2008; Williams et al., 2012; Henslee and Steele, 2018).

The mechanism of fluvastatin-induced apoptosis in P815 cells was similar to primary cells, with indications of mitochondrial damage. But unlike primary cells, we noted a rapid increase in surface c-Kit expression following fluvastatin exposure. Because mutant c-Kit activates MAPK, Akt, and other survival signals (Agarwal et al., 2015), it was surprising that even with a twofold increase in c-Kit expression, ERK and AKT activation was consistently suppressed by fluvastatin. We used three different ERK and AKT inhibitors to test the importance of these pathways. Although ERK blockade consistently induced BMMC apoptosis, only one of the three inhibitors induced P815 cell death under the same conditions. By contrast, all three Akt inhibitors elicited P815 apoptosis. These results suggest that Akt blockade may be a potent death-inducing fluvastatin effect in transformed mast cells, whereas ERK suppression may explain death in primary mast cells. Although we do not fully understand the divergence of Kit-mediated signaling in primary and transformed mast cells, these data suggest that Akt blockade may be effective against mastocytomas.

P815 cells also demonstrated a transient G2/M arrest coupled with synthesis phase reduction. These effects were distinct from primary mast cells, which showed no cell cycle changes preceding apoptosis. G2/M cell cycle arrest has been documented in transformed human mastocytomas by others and may be a facet of their neoplastic phenotype. For example, Bandara et al. (2018) found G2 accumulation when sphingosine kinase 1 was inhibited. Peter et al. (2018) noted similar effects with the multikinase inhibitor R763, which coincided with increased cell killing by midostaurin or dasatinib. Therefore, statins, which have an established history of safe use, could be considered as an adjuvant therapy for mast cell neoplasias.

In summary, we find that fluvastatin induces apoptosis in primary and transformed mast cells, likely because of a loss of c-Kit-mediated survival signals. Primary mast cells demonstrate a transient autophagy that may delay apoptosis, whereas transformed mast cells enter a G2/M cell cycle arrest preceding cell death. Because fluvastatin effects mapped to the geranylgeranyl pyrophosphate pathway involved in Ras family protein localization, our hypothesis is that statins reduce Ras family protein access or function, with subsequent loss of downstream survival signals from ERK and Akt. The identity of the affected Ras family proteins warrants further study. For example, c-Kit is a potent activator of the (Kirsten rat Ras protein) KRas-PI3K-Akt pathway, which requires KRas geranylgeranylation (Castellano and Downward, 2011; Akula et al., 2016). Although we observed loss of Akt activation in P815 cells, fluvastatin did not suppress c-Kit-mediated Akt phosphorylation in BMMC. This suggests variation in the critical isoprenylation targets. Alongside studies showing reduced allergic disease among statin users (Alexeeff et al., 2007; Huang et al., 2011; Wang et al., 2018), our data support further study of statins and the isoprenoid pathway as a means to suppress mast cell survival where this is clinically beneficial.

\section{Authorship Contributions}

Participated in research design: Paez, Kolawole, Schwartz, Straus, Gewirtz, Martin, Ryan.

Conducted experiments: Paez, Kolawole, Taruselli, Ajith, Dailey, Kee, Haque, Barnstein, McLeod, Caslin, Kiwanuka, Fukuoka, Le, Straus, Martin.

Performed data analysis: All authors.

Wrote or contributed to the writing of the manuscript: All authors.

\section{References}

Agarwal S, Kazi JU, Mohlin S, Påhlman S, and Rönnstrand L (2015) The activation loop tyrosine 823 is essential for the transforming capacity of the c-Kit oncogenic mutant D816V. Oncogene 34:4581-4590.

Akula MK, Shi M, Jiang Z, Foster CE, Miao D, Li AS, Zhang X, Gavin RM, Forde SD, Germain G, et al. (2016) Control of the innate immune response by the mevalonate pathway. Nat Immunol 17:922-929.

Alexeeff SE, Litonjua AA, Sparrow D, Vokonas PS, and Schwartz J (2007) Statin use reduces decline in lung function: VA Normative Aging Study. Am J Respir Crit Care Med 176:742-747.

Bandara G, Muñoz-Cano R, Tobío A, Yin Y, Komarow HD, Desai A, Metcalfe DD, and Olivera A (2018) Targeting sphingosine kinase isoforms effectively reduces growth and survival of neoplastic mast cells with D816V-KIT. Front Immunol 9: 631.

Calabro A, Tai J, Allen SL, and Budman DR (2008) In-vitro synergism of m-TOR inhibitors, statins, and classical chemotherapy: potential implications in acute leukemia. Anticancer Drugs 19:705-712.

Carter MC, Metcalfe DD, and Komarow HD (2014) Mastocytosis. Immunol Allergy Clin North Am 34:181-196.

Caslin HL, Taruselli MT, Paranjape A, Kiwanuka K, Haque T, Chumanevich AP, Oskeritzian CA, and Ryan JJ (2018) The use of human and mouse mast cell and basophil cultures to assess type 2 inflammation. Methods Mol Biol 1799:81-92.

Castellano E and Downward J (2011) RAS Interaction with PI3K: more than just another effector pathway. Genes Cancer 2:261-274.

Castells M and Butterfield J (2019) Mast cell activation syndrome and mastocytosis: initial treatment options and long-term management. J Allergy Clin Immunol Pract 7:1097-1106.

Dai Y, Khanna P, Chen S, Pei XY, Dent P, and Grant S (2007) Statins synergistically potentiate 7-hydroxystaurosporine (UCN-01) lethality in human leukemia and myeloma cells by disrupting Ras farnesylation and activation. Blood 109: $4415-4423$

Dos Santos C, McDonald T, Ho YW, Liu H, Lin A, Forman SJ, Kuo YH, and Bhatia R (2013) The Src and c-Kit kinase inhibitor dasatinib enhances p53-mediated targeting of human acute myeloid leukemia stem cells by chemotherapeutic agents. Blood 122:1900-1913.

Elieh Ali Komi D and Bjermer L (2019) Mast cell-mediated orchestration of the immune responses in human allergic asthma: current insights. Clin Rev Allergy Immunol 56:234-247.

Fischetti F, Carretta R, Borotto G, Durigutto P, Bulla R, Meroni PL, and Tedesco F (2004) Fluvastatin treatment inhibits leucocyte adhesion and extravasation in 
models of complement-mediated acute inflammation. Clin Exp Immunol 135: 186-193.

Fujimoto M, Oka T, Murata T, Hori M, and Ozaki H (2009) Fluvastatin inhibits mast cell degranulation without changing the cytoplasmic Ca2+ level. Eur J Pharmacol 602:432-438.

Henslee AB and Steele TA (2018) Combination statin and chemotherapy inhibits proliferation and cytotoxicity of an aggressive natural killer cell leukemia. Biomark Res 6:26.

Huang CC, Chan WL, Chen YC, Chen TJ, Chou KT, Lin SJ, Chen JW, and Leu HB (2011) Statin use in patients with asthma: a nationwide population-based study. Eur J Clin Invest 41:507-512.

Jin Y, Ding K, Wang D, Shen M, and Pan J (2014) Novel thiazole amine class tyrosine kinase inhibitors induce apoptosis in human mast cells expressing D816V KIT mutation. Cancer Lett 353:115-123.

Kambe N, Kambe M, Kochan JP, and Schwartz LB (2001) Human skin-derived mast cells can proliferate while retaining their characteristic functional and protease phenotypes. Blood 97:2045-2052.

Karlberg M, Ekoff M, Labi V, Strasser A, Huang D, and Nilsson G (2010) Proapoptotic Bax is the major and Bak an auxiliary effector in cytokine deprivationinduced mast cell apoptosis. Cell Death Dis 1:e43.

Khush KK and Waters DD (2006) Effects of statin therapy on the development and progression of heart failure: mechanisms and clinical trials. J Card Fail 12 664-674.

Kolawole EM, McLeod JJ, Ndaw V, Abebayehu D, Barnstein BO, Faber T, Spence AJ, Taruselli M, Paranjape A, Haque TT, et al. (2016) Fluvastatin suppresses mast cell and basophil IgE responses: genotype-dependent effects. J Immunol 196:1461-1470.

Metcalfe DD, Pawankar R, Ackerman SJ, Akin C, Clayton F, Falcone FH, Gleich GJ, Irani AM, Johansson MW, Klion AD, et al. (2016) Biomarkers of the involvement of mast cells, basophils and eosinophils in asthma and allergic diseases. World Allergy Organ J 9:7.

Metcalfe DD, Peavy RD, and Gilfillan AM (2009) Mechanisms of mast cell signaling in anaphylaxis. J Allergy Clin Immunol 124:639-646, NaN-648.

Möller C, Alfredsson J, Engström M, Wootz H, Xiang Z, Lennartsson J, Jönsson JI, and Nilsson G (2005) Stem cell factor promotes mast cell survival via inactivation of FOXO3a-mediated transcriptional induction and MEK-regulated phosphorylation of the proapoptotic protein Bim. Blood 106:1330-1336.

Morii E, Ito A, Jippo T, Koma Y, Oboki K, Wakayama T, Iseki S, Lamoreux ML, and Kitamura Y (2004) Number of mast cells in the peritoneal cavity of mice: influence of microphthalmia transcription factor through transcription of newly found mast cell adhesion molecule, spermatogenic immunoglobulin superfamily. Am J Pathol 165:491-499.

Niwa S, Totsuka T, and Hayashi S (1996) Inhibitory effect of fluvastatin, an HMGCoA reductase inhibitor, on the expression of adhesion molecules on human monocyte cell line. Int J Immunopharmacol 18:669-675.

Parikh A, Childress C, Deitrick K, Lin Q, Rukstalis D, and Yang W (2010) Statin induced autophagy by inhibition of geranylgeranyl biosynthesis in prostate cancer PC3 cells. Prostate 70:971-981.

Peter B, Bibi S, Eisenwort G, Wingelhofer B, Berger D, Stefanzl G, Blatt K, Herrmann H, Hadzijusufovic E, Hoermann G, et al. (2018) Drug-induced inhibition of phosphorylation of STAT5 overrides drug resistance in neoplastic mast cells. Leukemia 32:1016-1022.
Ramachandran R and Wierzbicki AS (2017) Statins, muscle disease and mitochondria. J Clin Med 6:75.

Roberts RF, Tang MY, Fon EA, and Durcan TM (2016) Defending the mitochondria: the pathways of mitophagy and mitochondrial-derived vesicles. Int $J$ Biochem Cell Biol 79:427-436.

Robinson AJ, Kashanin D, O'Dowd F, Fitzgerald K, Williams V, and Walsh GM (2009) Fluvastatin and lovastatin inhibit granulocyte macrophage-colony stimulating factor-stimulated human eosinophil adhesion to inter-cellular adhesion molecule-1 under flow conditions. Clin Exp Allergy 39:1866-1874.

Sassano A, Katsoulidis E, Antico G, Altman JK, Redig AJ, Minucci S, Tallman MS, and Platanias LC (2007) Suppressive effects of statins on acute promyelocytic leukemia cells. Cancer Res 67:4524-4532.

Siekmeier R, Lattke P, Mix C, Park JW, and Jaross W (2001) Dose dependency of fluvastatin pharmacokinetics in serum determined by reversed phase HPLC. $J$ Cardiovasc Pharmacol Ther 6:137-145.

Springer MZ and Macleod KF (2016) In brief: mitophagy: mechanisms and role in human disease. J Pathol 240:253-255.

Takai Y, Sasaki T, and Matozaki T (2001) Small GTP-binding proteins. Physiol Rev 81:153-208.

Tsujimura T, Furitsu T, Morimoto M, Isozaki K, Nomura S, Matsuzawa Y, Kitamura Y, and Kanakura Y (1994) Ligand-independent activation of c-kit receptor tyrosine kinase in a murine mastocytoma cell line P-815 generated by a point mutation. Blood 83:2619-2626.

Ushio H, Ueno T, Kojima Y, Komatsu M, Tanaka S, Yamamoto A, Ichimura Y, Ezaki J, Nishida K, Komazawa-Sakon S, et al. (2011) Crucial role for autophagy in degranulation of mast cells. J Allergy Clin Immunol 127:1267-1276.e6.

Wang JY, Yao TC, Tsai YT, Wu AC, and Tsai HJ (2018) Increased dose and duration of statin use is associated with decreased asthma-related emergency department visits and hospitalizations. J Allergy Clin Immunol Pract 6:1588-1595.e1.

Wang M and Casey PJ (2016) Protein prenylation: unique fats make their mark on biology. Nat Rev Mol Cell Biol 17:110-122.

Westerberg CM, Hägglund H, and Nilsson G (2012) Proteasome inhibition upregulates Bim and induces caspase-3-dependent apoptosis in human mast cells expressing the Kit D816V mutation. Cell Death Dis 3:e417.

Williams AB, Li L, Nguyen B, Brown P, Levis M, and Small D (2012) Fluvastatin inhibits FLT3 glycosylation in human and murine cells and prolongs survival of mice with FLT3/ITD leukemia. Blood 120:3069-3079.

Xenos ES, Stevens SL, Freeman MB, Cassada DC, and Goldman MH (2005) Nitric oxide mediates the effect of fluvastatin on intercellular adhesion molecule-1 and platelet endothelial cell adhesion molecule-1 expression on human endothelial cells. Ann Vasc Surg 19:386-392.

Youle RJ and Narendra DP (2011) Mechanisms of mitophagy. Nat Rev Mol Cell Biol 12:9-14.

Zhang J, Yang Z, Xie L, Xu L, Xu D, and Liu X (2013) Statins, autophagy and cancer metastasis. Int $J$ Biochem Cell Biol 45:745-752.

Address correspondence to: Dr. John J. Ryan, Department of Biology, Virginia Commonwealth University, Box 842012, Richmond, VA 23284-2012. E-mail: jjryan@vcu.edu 